\title{
Evaluation of enzyme immunoassay based on detection of pLDH antigen for the diagnosis of malaria
}

\author{
Khare $V^{1}$, Shukla $P^{2}$, Ansari $A^{3}, \operatorname{Yaqoob} S^{4}, \operatorname{Begum~} R^{5}$ \\ ${ }^{1}$ Dr. Vineeta Khare, Associate Professor, ${ }^{2}$ Dr. Priyanka Shukla, Assistant Professor, ${ }^{3}$ Dr. Ayesha Ansari, Junior Resident, \\ ${ }^{4}$ Dr. Shadma Yaqoob, Associate Professor, ${ }^{5}$ Dr. Rehana Begum, Professor and Head of Department, all authors are \\ affiliated with Department of Microbiology, Era's Lucknow Medical Collge and Hospital, Lucknow, U.P., India.
}

Address for correspondence: Dr. Vineeta Khare, Email: vinitakhare@yahoo.com

\begin{abstract}
Introduction: Timely diagnosis of malaria is a challenge in most endemic areas due to lack of resources. The methods most commonly used are microscopy, regarded as the gold standard, and rapid dipstick tests (RDT) which detect antigens in blood. Enzyme-Linked ImmunoSorbent Assay (ELISA) based tests are fast and easy to perform especially when large number of samples have to be tested. $\mathrm{p}$-LDH is a highly sensitive marker of malaria in blood The present study was done to assess the diagnostic performance of a p-LDH based ELISA on samples from clinically suspected malaria patients. Methods: We tested the sensitivity and specificity of a pLDH based, commercially available ELISA kit on both microscopy positive and negative samples. Microscopy was done for all suspected malaria patients and of these 146 samples ( 73 positive and 73 negative) were tested by the ErbaLisa PAN (LDH) malaria ELISA kit as well SD Bioline malaria antigen test (RDT) based on detection of both HRP-2 and p-LDH common to all four species. Results: The sensitivity of Elisa was $95.9 \%$ while specificity was $93.2 \%$ compared to gold standard microscopy while RDTs had $91.8 \%$ sensitivity and $86.3 \%$ specificity. All 67 samples positive by both microscopy and RDT were also positive by ELISA. Conclusion: p-LDH based ELISA promises to be a cost effective and reliable option for diagnosis of malaria in endemic areas like India.
\end{abstract}

Key words: Plasmodium, Enzyme-Linked Immunosorbent Assay, Sensitivity Specificity, pLDH

\section{Introduction}

Malaria is a vector borne disease caused by Plasmodium species and transmitted by female anopheles mosquitoes. In 2015, approximately 3.2 billion people - nearly half of the world's population were at risk of malaria. Nearly 300-400 million clinical episodes occur worldwide claiming lives of 1.5-2 million people each year. Currently more than $80 \%$ of the population of India lives in malaria risk areas [1]. In the face of increasing drug resistance, there is a need of rational and timely treatment of malaria. This in turn depends on the accuracy of malaria diagnosis.

Malaria presents a diagnostic challenge in most resource poor countries where this disease is endemic. Diagnosis made on clinical grounds is often inaccurate

Manuscript received $24^{\text {th }}$ September 2016

Reviewed: $4^{\text {th }}$ October 2016

Author Corrected: $15^{\text {th }}$ October 2016

Accepted for Publication $29^{\text {th }}$ October 2016 as symptoms are very nonspecific and overlap those of other febrile illnesses. The accepted gold standard for diagnosis is the examination of Giemsa stained thick blood smears. However, diagnosis by microscopy is not easy due to problems of variable parasitemia, sequestration of parasites in later stages of development in case of P. falciparum, pretreatment of cases, mixed infections and technical expertise required for microscopy [2].

Newer approaches to malaria diagnosis include the use of fluorescent stains like Acridine orange, PCR based detection, automated blood cell analysis and Quantitative Buffy Coats but these tests are not easy and require expensive equipments. The new generation antigen capture tests for malaria diagnosis are available as rapid dipstick tests (RDT). These tests are based on detection of HRP-2 antigen produced by $P$. falciparum, 
adolase and parasite lactate dehydrogenase (p-LDH) produced by all four common species of Plasmodium. These tests have become popular due to their simplicity, easy availability and lack of technical expertise in interpretation. Persistent HRP-2 antigenemia, pLDH production by gametocytes and, cross-reactivity with rheumatoid factor and heterophile antibodies are some of the causes of false positive RDTs. On the other hand false negative results may be caused by deletion or mutation of the hrp-2 gene or by anti-HRP-2 antibodies [3].

There are no commercially available molecular assays for malaria diagnosis and currently available molecular assays like PCR and LAMP are currently available in reference laboratories and are being used mainly for research or epidemiological purposes [4].

ELISA based tests are easy, fast and generally have good sensitivity and specificity. In ELISA format a large number of samples can be tested together. Antigen detection based on ELISA promises to be a precise, reproducible and cost-effective option for diagnosis of malaria in endemic areas like India [5].

\section{Aims and objectives}

Keeping in mind the seriousness of the disease, need for its early diagnosis and the limitations of available diagnostic techniques, the present study was undertaken to evaluate the diagnostic performance of an ELISA test based on $\mathrm{p}-\mathrm{LDH}$.

\section{Material and Methods}

The present study was an observational study conducted at the department of Microbiology, Era's Lucknow Medical College and Hospital, Lucknow for a period of one year. The study population comprised of all suspected malaria patients.

They included patients in all age groups visiting the hospital OPD, or admitted with history of fever of 2-3 days duration with any of the following symptoms: chills and rigor, splenomegaly, hepatomegaly, headache or abdominal discomfort. Patients already on antimalaria therapy and those unwilling to participate were excluded from the study.

Out of the study population, blood samples were collected at the time of first hospital visit. Thick and thin smears were prepared directly from these samples and rest of the sample was divided in 2 equal parts for ELISA and RDT.

Microscopy: Thick and thin blood films were prepared from the venous blood. Using a micropipette, $6 \mu \mathrm{L}$ of blood was placed on a clean slide for the thick smear and $4 \mu \mathrm{L}$ of blood for the thin smear. Two such slides were made from each sample. The slides were stained by Leishman stain and read by independently by two microscopists.

The thick films were screened for 200 oil-immersion fields ( $\times 100$ oil immersion objective) before declaring a film to be negative. If positive, parasite species were determined using thin smears.

ELISA: The ELISA kit evaluated in this study was ErbaLISA PAN (LDH) Malaria assay which is a commercial ELISA test kit designed for qualitative detection of $\mathrm{p}-\mathrm{LDH}$ antigen in whole blood. Microtitre wells were precoated with monoclonal antibodies to LDH of Plasmodium species. One hundred microliters of the sample diluent was transferred to blank and sample wells while 100 ul of controls were put in control wells.

This was followed by addition of $10 \mathrm{ul}$ sample and incubation for 45 minutes at $37^{\circ} \mathrm{C}$. Subsequently, the plates were washed six times with the 350 ul washing solution, and $100 \mu \mathrm{L}$ of the diluted Ab-conjugate was added to each well. After further incubation for 30 minutes, the plates were once again washed and $50 \mu \mathrm{L}$ of the TMB chromogen was added.

The plates were incubated for another 15 minutes in the dark, at room temperature and $100 \mu \mathrm{L}$ of the stopping solution was added. Spectrophotometric analysis was performed with an ELISA plate reader at an absorbance of $450 / 620 \mathrm{~nm}$.

RDT: SD Bioline malaria antigen test (RDT) based on detection of both HRP-2 and p-LDH common to all four species. Testing done on whole blood following manufacturer's instructions.

Data Analysis- For the performance characteristics, the following values were used: true positive (TP), false positive (FP), true negative (TN), and false negative (FN). Sensitivity, specificity, positive predictive value (PPV), negative predictive value (NPV) were calculated. 


\section{Results}

The present study was conducted in the department of Microbiology at Era's Lucknow Medical College and Hospital, Lucknow. A total of 146 cases of suspected malaria fulfilling the inclusion criteria were included in the study. Of these, 73 were malaria positive by microscopy while rest 73 were malaria negative. Malaria was confirmed by microscopy in these patients using thick and thin smears stained by Leishman stain. Of these, 9 cases were identified as P. falciparum while 64 cases were diagnosed as P. vivax. Equal number of positive and negative samples were selected to ensure proper evaluation of ELISA test on both malaria positive and negative samples.

The age of patients included in the study ranged from 6 to 80 years with a mean age of 35.8 years. Out of 146 patients included in the study, male: female ratio was 1:0.97 with 74 males and 72 females. Comparison of microscopy and ELISA results for these patients are presented in table 1 while comparison of microscopy and RDT results for these patients are presented in table 2 .

Table- 1: Comparison of microscopy and ELISA results.

\begin{tabular}{|c|c|c|c|}
\hline Microscopy results & Elisa positive & Elisa negative & Total \\
\hline Positive & 70 & 3 & 73 \\
\hline Negative & 5 & 68 & 73 \\
\hline
\end{tabular}

Thus sensitivity of Elisa was $95.9 \%$ while specificity was $93.2 \%$ compared to gold standard microscopy.

Table-2: Comparison of microscopy and RDT results.

\begin{tabular}{|c|c|c|c|}
\hline Microscopy Results & RDT Positive & RDT Negative & Total \\
\hline POSITIVE & 67 & 6 & 73 \\
\hline NEGATIVE & 10 & 63 & 73 \\
\hline
\end{tabular}

Thus sensitivity of RDT was $91.8 \%$ while specificity was $86.3 \%$ compared to gold standard microscopy. All 67 samples positive by both microscopy and RDT were also positive by ELISA.

Table-3: Sensitivity and Specificity of both ELISA and RDT compared to Microscopy.

\begin{tabular}{|c|c|c|c|c|c|c|c|c|c|}
\hline Test & TP & FN & TN & FP & $\begin{array}{c}\text { SENS } \\
(\%)\end{array}$ & $\begin{array}{c}\text { SPEC } \\
(\boldsymbol{\%})\end{array}$ & PPV & $\begin{array}{c}\text { NPV } \\
\text { Accuracy } \\
(\boldsymbol{\%})\end{array}$ \\
\hline ELISA & 70 & 3 & 68 & 5 & 95.9 & 93.2 & 93.3 & 95.8 & 94.5 \\
\hline RDT & 67 & 6 & 63 & 10 & 91.8 & 86.3 & 87 & 91.3 & 89.7 \\
\hline$\chi^{2}$ & \multicolumn{2}{|c|}{1.066} & \multicolumn{2}{|c|}{1.858} & & & & & 2.313 \\
\hline $\mathrm{p}$ & \multicolumn{2}{|c|}{0.302} & & & & & 0.128 \\
\hline
\end{tabular}

TP=True positive, FN=False Negative, TN=True Negative, FP=False Positive, SENS= Sensitivity, SPEC=Specificity, $\mathrm{PPV}=$ Positive Predictive Value, NPV=Negative Predictive Value.

All 9 P.falciparum cases were correctly identified as positive by all the 3 tests used. Six $P$. vivax positive were missed by RDT and 3 of them also by ELISA. Out of 10 false positive $P$. vivax results by RDT, one was also positive by ELISA.

\section{Discussion}

Rational treatment of malaria is needed to avoid the overuse of antimalarials, delay the development of drug resistance, save cost and improve treatment outcomes. Timely and accurate diagnosis is the only way of ensuring effective and rational therapy. Microscopy and rapid diagnostic tests (RDTs), represent the two most commonly used tests today employed for diagnosing malaria.

The sensitivity of microscopy depends on a number of factors like the quality of smear and staining, experience of the microscopist, magnitude of 
parasitemia, and number of fields observed. Poor blood film preparation generates artifacts which can be easily mistaken for malarial parasites. Similarly bacteria, fungi, stain precipitation, dirt or cell debris and normal blood components such as platelets may be mistakenly reported as malaria. The chance of false negative results increases with decreasing parasite densities. Errors in species identification are also commonly seen at low parasite densities. Experience of microscopist and time/number of microscopic fields examined reduces such errors [6]. False positive results lead to misuse and thus decreased efficacy of anti-malarial drugs, while false negative results could lead to suboptimal treatment and poor prognosis of patients. Moreover it decreases the specificity of new diagnostics tests under evaluation.

In the present study, compared to microscopy 10 false positive results were seen with RDTs and one of these was also positive by ELISA. Due to above mentioned shortcomings of microscopy, it is difficult to know whether or not these cases were true negatives. Molecular tests like PCR could have been helpful but these tests are not available at most centers including ours.

The need for an easy diagnostic test which can be done rapidly and is not dependent on experienced staff, led to the development of rapid tests. Rapid diagnostic test is a device based on immunochromatography which uses monoclonal antibodies directed against the target parasite antigens. These antigens are impregnated on a test strip and a small amount of blood, usually 5-15 $\mu 1$, is used for these tests which give results, usually a colored test line, in 5-20 minutes. RDTs, do not require much infrastructure or investment and are easy to interpret without much past experience. Commercial tests using different combinations of target antigens that can distinguish $P$. falciparum from the three nonfalciparum species are available. Therefore RDT consumption, especially in developing countries, has increased for the past few years.

RDT tests in general have shown good sensitivity and specificity and agreement to the reference light microscopy and thus RDT consumption, especially in developing countries, has increased for the past few years [8]. However, RDTs generally miss about five percent of cases and RDTs which aim to identify 'nonfalciparum malaria only' as a proxy for P. vivax may miss between $11 \%$ to $22 \%$ of cases. In addition, such tests do not allow the identification of non-falciparum malaria as part of a mixed infection, or the differentiation of $\mathrm{P}$. vivax from $\mathrm{P}$. ovale and P. malariae [9]. Persistent HRP-2 antigenemia, pLDH production by gametocytes and, cross-reactivity with rheumatoid factor and heterophile antibodies are some of the causes of false positive RDTs [10]. On the other hand false negative results may be caused by deletion or mutation of the hrp-2 gene or by anti-HRP-2 antibodies [3].

Enzyme-linked immunosorbent assays (ELISAs) tests provide a fast, relatively inexpensive, and reliable way to detect malaria. It allows for the testing of large numbers of samples within a short time frame and thus can be useful for blood bank screening. ELISA may serve as a suitable adjunct to microscopy. There is a need of commercial species-specific ELISA tests kits.

In present study, overall detection rate for ELISA was $51.4 \%$ where as for RDT it was $52.7 \%$. As present study used a purposive sampling design in which $50 \%$ were microscopy positive and 50\% microscopy negative cases thus overall detection rate more than $50 \%$ indicated a definitive false positive pattern for both diagnostic techniques. Akotet et al. (2014) also observed a higher detection rate than microscopy in their assessment of SD Bioline malaria Ag-Pf/Pan RDT test (11). A false positivity is indicative of reduction in specificity. However actual efficacy of two methods could only be evaluated with their respective performance against microscopy.

In this study, compared to microscopy, ELISA had sensitivity, specificity, PPV and NPV of 95.9\%, 93.2\%, 93.3\% and $95.8 \%$ respectively. RDT had a sensitivity, specificity, PPV and NPV of 91.8\%, 86.3\%, 87\% and 91.3\% respectively .These findings indicated that RDT as compared to ELISA despite having higher detection rate in suspected cases had a lower sensitivity as well as specificity which might be attributed to a higher number of both false positive as well as false negative cases in this technique as compared to ELISA. With respect to accuracy too ELISA scored better with an accuracy of $94.5 \%$ compared to $89.7 \%$ of RDT but this difference was not statistically significant.

Most previous studies have found antigen based ELISA to be more sensitive and more specific compared to ELISA. Noedl H et al. found overall sensitivity of the HRP2 ELISA for P. falciparum malaria to be $98.8 \%$ $(95 \% \mathrm{CI}, 93.6-100 \%)$ and the specificity was $100 \%$ 
(95\% [12]. The sensitivity and specificity of the ELISAbased NovaLisa test, based on antibody detection of Plasmodium spp. using malarial-specific antigens (MSP1, CSP, and a chimeric multi-epitope antigen from P. falciparum and P. vivax), was 89.0 vs $91.6 \%$, respectively compared with the microscopy [13].

Studies where ELISA performance was found insufficiently sensitive were mostly antibody based and these tests were found to have little role even for blood screening of P.vivax [14]. The combined use of antigen and antibody ELISAs has been reported to improve the diagnostic sensitivity of P. vivax. cases [15].

The present study was limited by purposive sampling designs and small sample size, further studies with larger sample size in cross section of suspected malarial cases are highly recommended. Also, the present study had more $P$. vivax cases $(87.7 \%)$ and thus this $\mathrm{pLDH}$ based ELISA needs to be evaluated on more P.falciparum cases.

In present study detection rate of ELISA as well as RDT was $100 \%$ for microscopically positive P. falciparum however for microscopically positive P.vivax was $90.6 \%$ for RDT and for ELISA 95.3\%. The findings in this study show that ELISA as well as RDT have a great clinical importance from management point of view. Although both techniques had high sensitivity and specificity, ELISA had overall better sensitivity as well as specificity.

\section{Conclusion}

For rapid diagnosis of malaria, ELISA based antigen detection test could be a reliable alternative to RDTs especially with large sample loads. They can be reliable adjunct to microscopy in malaria diagnosis.

\section{Funding: Nil, Conflict of interest: None. Permission of IRB: Yes}

\section{References}

1. WHO: WORLD MALARIA REPORT 2015 Country profile India 2015 [http://www.who.int/malaria/ publications/country-profiles/profile_ind_en.pdf?ua=1] last accessed on $10^{\text {th }}$ October, 2015.

2. Tangpukdee N, Duangdee C, Wilairatana P, Krudsood S. Malaria diagnosis: a brief review. Korean J Parasitol. 2009 Jun; 47(2):93-102. doi: 10.3347/kjp. 2009. 47.2.93. Epub 2009 May 26.
3. Zurovac D, Midia B, Ochola SA, English M, Snow RW. Microscopy and outpatient malaria case management among older children and adults in Kenya. Trop Med Int Health. 2006 Apr;11(4):432-40.

4. Britton S, Cheng Q, McCarthy JS. Novel molecular diagnostic tools for malaria elimination: a review of options from the point of view of high-throughput and applicability in resource limited settings. Malar J. 2016 Feb; 15: 88. doi: 10.1186/s12936-016-1158-0.

5. Kifude CM, Rajasekariah HG, Sullivan DJ, Stewart VA, Angov E, Martin SK, Diggs CL, N. Waitumbi JN. Enzyme-Linked Immunosorbent Assay for Detection of Plasmodium falciparum Histidine-Rich Protein 2 in Blood, Plasma, and Serum. Clin Vaccine Immunol. 2008 Jun;15(6):1012-8. doi: 10.1128/CVI.00385-07.

6.Wongsrichanalai C, Barcus MJ, Muth S, Sutamihardja A, Wernsdorfer WA. A Review of Malaria Diagnostic Tools: Microscopy and Rapid Diagnostic Test (RDT) Am. J. Trop. Med. Hyg., 77 (Suppl 6); 2007:119-27.

7. Bell DR, Wilson DW, Martin LB, 2005. Falsepositive results of a Plasmodium falciparum histidinerich protein 2-detecting malaria rapid diagnostic test due to high sensitivity in a community with fluctuating low parasite density. Am J Trop Med Hyg 73: 199-203.

8. Moges B, Amare B, Belyhun Y, Tekeste Z, Gizachew M, Workineh M, Gebrehiwot A, Woldeyohannes D, Mulu A, Kassu A. Comparison of Care Start ${ }^{\mathrm{TM}}$ HRP2/pLDH COMBO rapid malaria test with light microscopy in north-west Ethiopia. Malar J. 2012 Jul;11:234. doi: 10.1186/1475-2875-11-234.

9. Abba K, Kirkham AJ, Olliaro PL, Deeks JJ, Donegan S, Garner P, Takwoingi Y. Rapid diagnostic tests for diagnosing uncomplicated non-falciparum or Plasmodium vivax malaria in endemic countries. Cochrane Database Syst Rev. 2014 Dec 18; (12): 1195. doi: 10.1002/14651858.CD011431.

10. Lee JH, Jang JW, Cho CH, Kim JY, Han ET, Yun SG, Lim CS. False-positive results for rapid diagnostic tests for malaria in patients with rheumatoid factor. $\mathbf{J}$ Clin Microbiol. 2014 Oct;52(10):3784-7. doi: 10. 1128/JCM.01797-14. Epub 2014 Jul 23.

11. Akotet BMK, Nkare CA, Mbouoronde OC, MawiliMboumba DP. Performances of SD Bioline Malaria Ag-P.F/Pan RDT for the Diagnosis of Malaria in Febrile Patients Living In Gabon, Central Africa. Malar 
Chemoth Cont Elimination.2014; 3:125. doi: 10. 4172/ 2470-6965.1000125.

12. Noedl H, Yingyuen K, Laoboonchai A, Fukuda M, Sirichaisinthop J, Miller RS. Sensitivity and specificity of an antigen detection ELISA for malaria diagnosis. Am J Trop Med Hyg. 2006 Dec;75(6):1205-8.

13. Thongdee P, Chaijaroenkul W, Kuesap J, NaBangchang K. Nested-PCR and a new ELISA-based NovaLisa test kit for malaria diagnosis in an endemic area of Thailand. Korean J Parasitol. 2014 Aug;52(4): 377-81. doi:10.3347/kjp.2014. 52.4.377. Epub 2014 Aug 29.
14. Oh JS, Kim JS, Lee CH, Nam DH, Kim SH, Park DW, Lee CK, Lim CS, Park GH. Evaluation of a malaria antibody enzyme immunoassay for use in blood screening. Mem Inst Oswaldo Cruz. 2008 Feb; 103 (1):75-8. Epub 2008 Jan 31.

15. Kim J, Jang JW, Kim JY, Oh DJ, Lim CS. Combined Use of Malaria Antigen and Antibody Enzyme-Linked Immunosorbent Assay for Blood Screening of Plasmodium vivax in the Republic of Korea. Med Princ Pract. 2016;25(3):212-8. doi:10. 1159/000444144.

\section{How to cite this article?}

Khare V, Shukla P, Ansari A, Yaqoob S, Begum R. Evaluation of enzyme immunoassay based on detection of pLDH antigen for the diagnosis of malaria. Int J Med Res Rev 2016;4(10):1897-1902.doi:10.17511/ijmrr. 2016.i10.28. 